\title{
Mathematical Modelling and Analysis of Corruption of Morals amongst Adolescents with Control Measures in Kenya
}

\author{
Nathan Oigo Mokaya $\left(\mathbb{D},{ }^{1}\right.$ Haileyesus Tessema Alemmeh, ${ }^{2}$ Cyrus Gitonga Ngari, ${ }^{3}$ \\ and Grace Gakii Muthuri ${ }^{4}$ \\ ${ }^{1}$ Department of Mathematics, Masinde Muliro University of Science and Technology, Kakamega, Kenya \\ ${ }^{2}$ Department of Mathematics, College of Natural and Computational Science, University of Gondar, Gondar, Ethiopia \\ ${ }^{3}$ Department of Mathematics, Computing and Information Technology, University of Embu, Embu, Kenya \\ ${ }^{4}$ Department of Mathematics, Meru University of Science and Technology, Meru, Kenya
}

Correspondence should be addressed to Nathan Oigo Mokaya; nathanoigo@yahoo.com

Received 17 November 2020; Revised 27 January 2021; Accepted 10 March 2021; Published 21 April 2021

Academic Editor: Chun Wei

Copyright (C) 2021 Nathan Oigo Mokaya et al. This is an open access article distributed under the Creative Commons Attribution License, which permits unrestricted use, distribution, and reproduction in any medium, provided the original work is properly cited.

\begin{abstract}
In the present paper, we formulate a new mathematical model for the dynamics of moral corruption with comprehensive ageappropriate sexual information and provision of guidance and counselling. The population is subdivided into three (3) different compartments according to their level of information on sexual matters. The model is proved to be both epidemiologically and mathematically well posed. The existence of unique morally corrupt-free and endemic equilibrium points is investigated. The basic reproduction number with respect to morally corrupt-free equilibrium is obtained using next generation matrix approach to monitor the dynamics of corrupt morals and ascertain its level in order to suggest effective intervention strategies to control this problem. The local as well as global asymptotic stability of these equilibrium points is studied. The analysis reveals a globally asymptotically stable morally corrupt-free equilibrium whenever $\mathscr{R}_{0} \leq 1$ and a globally asymptotically stable endemic equilibrium if otherwise. Further analysis, using center manifold theory, shows that the model exhibits forward bifurcation insinuating that the classical epidemiological requirement of $\mathscr{R}_{0} \leq 1$ is necessary and sufficient for elimination of moral corruption. A brief discussion on the graphical results using the available numerical procedures is shown. From numerical simulations, it was ascertain that integrated control strategy is the best approach to fight against moral corruption transmission. Lastly, some key parameters that show significance in the moral corruption elimination from the society are also exploited.
\end{abstract}

\section{Introduction}

While corruption can be defined as "abuse of public office for private gain," involving misappropriation of public authority for personal advantage [1], corruption of morals on the other hand is erosion of morals or deviating from societal way of life and civilization for personal gain disregarding the damages that come with it. Corruption of morals and materialistic corruption are one and the same since both entail the misuse of entrusted authority for personal advantage. The warped and brutal portrayal of sex in books, plays, music, magazines, and movies is corrupting a society and a civilization, and if not halted and reversed, it could poison the wellspring of Kenyan adolescents, their culture, and civilization. Moral corruption has become a malaise in Kenya. It is an important issue due to the potential adverse impact on the early pregnancies and child's health and to the large negative individual and social consequences that result from curtailment of the girls' education and societal loss of human resource. Corruption of morals increases adolescents' fertility rate in low and middle income countries such as Kenya which presents a severe impediment to development and can lead to school dropout, lost productivity, and the intergenerational transmission of poverty.

A survey by Kenya Demographic Health Survey (KDHS) 2014 [2] indicated that over 18 percent of adolescent girls 
between the age of 10-19 years are mothers in Kenya. Furthermore, in reference to United Nations Population Fund Report 2018 [3], Kenya recorded 378,397 adolescent pregnancies for girls aged 10-19 years between July 2016 and June 2017, and specifically, 28,932 girls aged 10-14 and 349,465 girls aged 15-19 became pregnant. Further, a research by Aids Control Council [3] reveals that girls aged between 15 and 24 years now account for the highest number of new infections. The group accounts for one-third of the 44,789 new HIV adult infections in 2017. National AIDs and STIs Control Programme (NASCOP) 2018 [4] estimates show that 105,230 adolescents between 10-19 years were living with $\mathrm{HIV}$; in addition, 184,700 young adults (15-24) are also infected. Sadly, the data indicates that adolescents account for one in every 10 AIDS-related deaths.

Approximately 10 percent of young people who are sexually active reported regular use of birth control and were aware of those birth control measures in Kenya [5]. These low number of contraception use are attributed to barriers like structural barriers such as cost, sociocultural barriers such stigma around adolescent sexuality, or even individual barriers such as limited or incorrect knowledge of Sexual and Reproductive Health (SRH). For instance, to use a birth control in Kenya, an adolescent must raise the issue with a possibly suspicious or resistant partner, hustle funds for supplies, overcome fear about rumored side effects, and bargain with a health system that is not accommodating to adolescent clients [6]. Alternatively, use of natural family planning can rescue some of these issues. However, it requires meticulous charting and mastery of the female menstrual cycle that few or none of young people have.

Kenya has enough policy infrastructure in place such as Constitution (2010) which expressly recognises in article 43 (1) that every person has the right to the highest attainable standard of health, which includes the right to health care services, including reproductive health care. In addition the country has enacted Children's Act 2001, Prohibition of Female Genitals Mutilation (FGM) Act (2011), Person with Disability Act (2003), Human Immunodeficiency Virus (HIV) and Acquired Immunodeficiency Syndrome (AIDS) Prevention and Control Act (2006), and Marriage Act (2014) all which provide the legal framework to support provision of Adolescent and Youth Friendly Services (AYFS). Other policies that support AYFS include Kenya Vision 2030, National Reproductive Health Policy (2007), National Youth Policy (2007), Gender Policy in Education (2007), Kenya Health Policy (2012-2030), the Education Sector Policy on HIV and AIDS (2013), National Adolescent Sexual Reproductive Health Policy (2015), and the National School Health Policy (2009) [7]. However, it has been identified that there is a paucity of strategic direction to address the problem of early child bearing and teenage pregnancy reflected in, on the one hand, ineffective implementation of the programmes to help adolescents protect themselves from early pregnancy and stay in school and, on the other hand, weak enforcement of the existing laws that protect teenage from abuse and exploitation. This has made it easier for this young generation to be easily swayed and corrupted morally.
Age appropriate is the suitability of information and services for people of a particular age bracket, particularly in relation to adolescent growth and development. Age-appropriate information entails culturally relevant approach to teaching about sexuality and relationships by providing scientifically accurate, realistic, and nonjudgmental information. Sexual information provides opportunities to young people to explore their values and attitudes and to build decision-making, communication skills, and risk reduction skills about many aspects of sexuality [7]. Moreover, it provides a structured opportunity for adolescents to gain knowledge and skills, to explore their altitudes and values, and to practice the decisionmaking and other life skills necessary for making healthy informed choices about their sexuality lives [8]. For sexual health to be attained and maintained, the sexual rights (see in [7]) of all persons must be respected, protected, and fulfilled.

Kenyan adolescents are experiencing social turmoil resulting from conflicting values as the country become more urbanized and industrialised [9]. In most ethnic groups, adolescence commenced with circumcision right of passage that marked transition from childhood to adulthood. Over this period, initiates were secluded from community while a selected teacher explained to them their roles in the society and they were taught about sexual behaviours, sexual relationships, gender roles, and pregnancy matters. Such customs conferred peer-group identity and promoted a social and personal sense of belonging [10]. Clear messages regarding sexual behaviours were conveyed to these young people. The society now is slowly deviating from the conservative traditional values and altitudes on safe sex discussion/education.

With the arrival of missionaries and colonists in Kenya in the 1800s and new political, social, and economic orders, most sexual socialization rituals were discarded with no alternative provided. Industrialization and urbanisation continue to alter the sociocultural structure of the traditional communities. Education functions, which formally rested within the family and communities, are increasingly being taken over by local and national governments, churches, and community groups. However, these leaders remain apprehensive and uncomfortable about policies and legislation related to youths, such as those affecting sex education, access to contraceptives, expulsion of pregnant girls from schools, and enforcement of regulations of age of consent and marriage. Efforts to address sexual and reproductive health matters are resisted by parents, religious leaders, and political leaders despite the mounting evidence that young people are initiating sex earlier than in the past. Proven interventions like appropriate sexuality education are often dismissed with the view that they would encourage young people to indulge in sex. Probably, the conservative nature and silence to factual information are the reason why we have high incidence of HIV, unhealthy sexual relationships, unsafe abortion, sexual abuse, and exploitation among the vulnerable adolescents.

The exposure of adolescents to these moral corrupt contents in secular music, dances, plays, and videos is the cause of the rampage teen pregnancies and immoral 
behaviours observed. Statistics have it that an average adolescent spends 3 to 4 hours per day watching television or in social media such YouTube, Facebook, and more. Furthermore, 83 percent of the programme most frequently watched contains some sexual content. Once these adolescents become addicted to such sexual content, they look for more potent, more explicit, and more graphic kinds of materials. They keep craving for something which is harder and gives them a greater sense of excitement until the point where the sexual content (pornography) only goes so far that jumping off point where they begin to think maybe actually doing (engage in sex) will give them that which is just beyond reading about it or looking at it.

A number of studies and research in social sciences have focused on this topic and other related topics more so on material corruption. However, the mathematical studies and research on this topic are still limited. Mathematical modeling is an important tool in comprehending the corruption transmission dynamics and in decision-making processes regarding intervention programmes necessary and sufficient for addressing corruption of morals. In the available literature, there are only a few quantitative studies on corruption. For instance, the study by [11] proposed and analysed a deterministic model for corruption in a population and concluded from his numerical simulations that corruption can only be reduced to a manageable level but not totally eradicated. In [12], the authors developed an SIR model for the corruption dynamics and extended the model to include optimal control with a single optimal control strategy. They concluded that the level of corruption in society can be curbed if efforts to control corruption are increased and put in place through media awareness and punishments.

The authors of [13] developed an epidemiological compartment model for corruption in Kenya, mainly considering political office holders and sympathisers. The study by [14] proposed a mathematical model for corruption by considering awareness created by anticorruption and counselling in jail. The existence of unique corruption-free and endemic equilibrium points was investigated, and the basic reproduction number was computed. It was also shown that the corruption endemic equilibrium is locally asymptotically stable provided that the basic reproduction number is greater than one. In [15], the author developed an epidemiology model for the corruption dynamics. Furthermore, he extended the model to include optimal control with combinations of two corruption preventive measures, namely, (i) campaigning about corruption through media and advertisement and (ii) exposing the corrupted individuals to jail and giving punishment. Tessema Alemneh [15] concluded that the combination of both strategies is effective in bringing down the exposed and corrupted population in the specified period of time.

Adeyemi $[1,16]$ developed a five-compartmental model to gain insight on the parameter that has the greatest impact on the transmission dynamics of corruption. The corruption-free equilibrium state, characteristic equation, eigenvalues of the corruption model and the reproduction number were obtained. The result from the research showed that the rate of effective corruption contact among the populace has the greatest impact. The studies by $[17,18]$ concluded that the higher the rate of public enlightenment against corruption, the lower the number of corrupt individuals and that mass education and religious teaching on corruption has significant impact on corrupttion reduction, respectively.

In this paper, a compartmental mathematical model was used to study the effectiveness of all possible combinations of two moral corruption preventive measures, namely, (i) provision of youth friendly services and comprehensive ageappropriate sexual education and (ii) provision of guidance and counselling services. Teenage sexual activities are assumed to be a nonstandard epidemic process that rarely emerges out of nothing but is usually related to some already sexually infected environment (such as uncontrolled media contents, misinformed generation, and naive society which is not willing to address the matter) which corrupt susceptible individuals morally.

The paper is organized as follows. In section 2, formulation of the model is done; in section 3 , analysis of the mathematical model is presented; and in section 4, we present some numerical simulations supporting our analytical results. The final section concludes the paper with a short summary of the model, recommendation of our model, and ideas for future studies.

\section{The Mathematical Model Formulation and Description}

The total population of adolescents $N(t)$ is divided into three compartments. Those adolescents who are susceptible to moral corruption are susceptible adolescents $S(t)$, those adolescents who are well informed and guided and can make informed decisions or choices are informed adolescents $I(t)$, and those adolescents who are performing or engaging in activities resulting to corruption of morals are morally corrupt adolescents $C(t)$, at time $t>0$. We assume that there is a positive recruitment $\Lambda$ into the susceptible class by birth or immigration. Out of this class, $\gamma$ proportion will join the informed subpopulation which never involves in moral corruption practices irrespective of the circumstances around them. We consider a positive exit rate $\mu$ (the rate at which the adolescents graduate from the 10-19 years age bracket) for all adolescents at all time under the study. Susceptible adolescents will have a contact rate with the morally corrupted individuals at rate $\Pi$ with a corruption transmission probability $\theta$ per contact and moved to morally corrupt class. The morally corrupted individuals get informed about the effect of moral corruption through youth friendly programmes and guidance and counselling at religion level, schools, and through mass media and move to the informed subpopulation at a rate of $\omega$, where $(\omega=\delta+\alpha+\sigma)$. All the descriptions of the parameters are listed in Table 1. $S(t), C(t)$, and $I(t)$ are variables that represent numbers of the adolescents in the three compartments at time $t$ in years. With reference to the above considerations, we have the compartmental flow diagram 
TABLE 1: Description of parameters of moral corruption model (1).

\begin{tabular}{lc}
\hline Parameter & Description \\
\hline$\Lambda$ & Recruitment rate into adolescent group \\
$\delta$ & Natural recovery rate (self-change) of individuals \\
$\alpha$ & Rate of guidance and counselling \\
$\mu$ & Exit rate (the rate at which the adolescents graduate from the 10-19 years age bracket) \\
$\sigma$ & Measure the availability level of adolescent friendly reproductive health services and sexual information \\
$\theta$ & Moral corruption transmission probability per contact \\
$\Pi$ & Contact rate \\
$\gamma$ & Rate at which susceptible individuals become immune to moral corruption \\
\hline
\end{tabular}

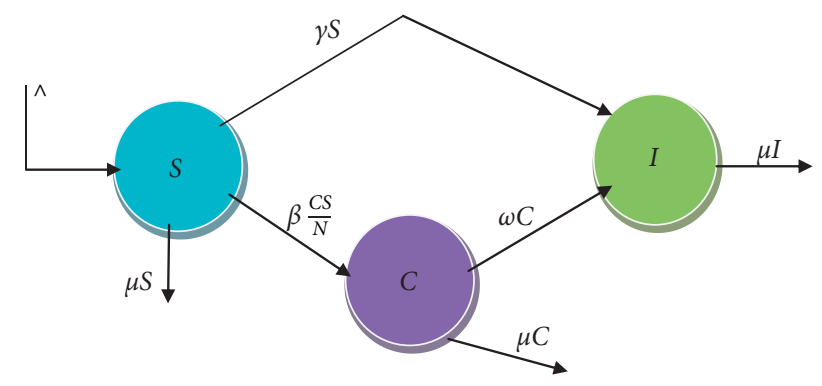

Figure 1: Schematic diagram of the corruption transmission dynamic model.

shown in Figure 1. From the flow chart, the model will be governed by the following system of differential equations.

$$
\left\{\begin{array}{l}
\frac{\mathrm{d} S}{\mathrm{~d} t}=\Lambda-\beta \frac{C(t) S(t)}{N(t)}-K_{1} S(t), \\
\frac{\mathrm{d} C}{\mathrm{~d} t}=\beta \frac{C(t) S(t)}{N(t)}-K_{2} C(t), \\
\frac{\mathrm{d} I}{\mathrm{~d} t}=K_{3} S(t)+K_{4} C(t)-\mu I(t),
\end{array}\right.
$$

with the initial conditions

$$
\begin{gathered}
S(0)=S_{0}>0, \\
C(0)=C_{0} \geq 0, \\
I(0)=I_{0} \geq 0,
\end{gathered}
$$

where $K_{1}=\gamma+\alpha+\sigma+\mu, \quad K_{2}=\delta+\alpha+\sigma+\mu$, $K_{3}=\gamma+\alpha+\sigma, K_{4}=\delta+\alpha+\sigma$, and $\beta=\Pi \theta$. The parameter $0<\sigma<1$ measures the rate change of corruption of morals due to availability of youth friendly services and information on age-appropriate sexual matters and $0<\alpha<1$ measures the rate change of corruption of morals due guiding and counselling (that is, at religion, home, or school level).

\section{Model Analysis}

3.1. Positivity of Solutions and Invariant Region. Since model (1) monitors human population, it is necessary that all its state variables and associated parameters be positive for future time. This will be established by the following proposition.
Proposition 1. Under the flow described by (1), the region

$$
\Gamma=\left\{(S, C, I) \in \mathscr{R}_{\geq 0}^{3}: S+C+I \leq \frac{\Lambda}{\mu}\right\},
$$

is positively invariant and attracting within $\mathscr{R}_{\geq 0}^{3}$ (i.e., all solutions starting in $\Gamma$ remain in $\Gamma$ for all $t>0$, while solutions starting outside $\Gamma$ either enter or approach in the limit). For detailed proof, see Lemma (1) and (2) in [19]. Thus, model (1) is epidemiologically and mathematically well posed in the region $\Gamma$ and can be analysed.

\subsection{Moral Corruption-Free Equilibrium Point (MCFEP)}

Definition 1. (equilibrium solution). Let $x^{*}, f \in \mathscr{R}^{n}$, then $x^{*}$ is an equilibrium or steady-state solution of the dynamical system defined by the autonomous system of firstorder ordinary differential equations in (1) if $f\left(x^{*}\right)=0$ [20].

Definition 2. (morally corrupt-free equilibrium solution). A morally corrupt-free or infection-free equilibrium solution exists when there is no moral corruption in the population. The trivial equilibrium solution $E^{0}$ exists and is the only equilibrium solution for $\mathscr{R}_{0} \leq 1$. The moral corruption-free equilibrium point of model (1) is obtained by equating all equations of model (1) to zero and letting $C=0$. Model (1) has an MCFEP, given by

$$
E^{0}\left(S_{0}, 0, I_{0}\right)=\left(\frac{\Lambda}{K_{1}}, 0, \frac{K_{3} \Lambda}{\mu K_{1}}\right) .
$$

3.3. Basic Reproduction Number. The linear stability of $E^{0}$ can be established using the next generation operator method on system (1). The matrices F (for the new infection terms) and V (of the transition terms) are given, respectively, as follows:

$$
\begin{aligned}
& F=\left\{\begin{array}{cc}
\frac{\beta \mu}{K_{1}} & 0 \\
0 & 0
\end{array}\right\}, \\
& V=\left\{\begin{array}{cc}
K_{2} & 0 \\
-K_{4} & \mu
\end{array}\right\} .
\end{aligned}
$$


It follows then that the associated reproduction number, denoted by $\mathscr{R}_{0}=\rho\left(\mathscr{F} \mathscr{V}^{-1}\right)$, is given by the following:

$$
\mathscr{R}_{0}=\frac{\mu \beta}{K_{1} K_{2}}
$$

where $\rho$ is the spectral radius (dominant eigenvalue in magnitude) of the next generation matrix, $F V^{-1}$. Using the parameter values from Table 2 , different values of $\mathscr{R}_{0}$ are given in Tables 3-5. Hence, using Theorem 1 of [19], the following result stated in Lemma 1 is established.

\subsection{Local Stability of MCFEP}

Lemma 1. The morally corrupt-free equilibrium, $E_{0}$, of model (1) is locally asymptotically stable (LAS) (see appendix A for definition of LAS) if $\mathscr{R}_{0} \leq 1$ and unstable if $\mathscr{R}_{0}>1$. The threshold quantity $\mathscr{R}_{0}$ is the reproduction number of the corrupt morals [21]. It represents the average number of young people at puberty; one morally corrupt person can infect morally in a purely susceptible population throughout the contact period. The basic reproduction number $\mathscr{R}_{0}$ is essential in the sense that it gives the insight where control strategies should be employed. Generally, the corrupt morals disappear from the community if $\mathscr{R}_{0}<1$ and may establish itself if $\mathscr{R}_{0}>1$. Thus, $\mathscr{R}_{0}$ serves as a direct quantitative measure of the intervention effort needed to eradicate the corrupt morals from the community. The critical case $\mathscr{R}_{0}=1$ represents the situation in which the corrupt morals reproduce itself thereby leaving the community with a similar number of morally corrupt cases at any time $\mathscr{R}_{0}>1$.

Theorem 1. The unique morally corrupt-free equilibrium $E_{0}=\left(\left(\Lambda / K_{1}\right), 0,\left(K_{3} \Lambda / \mu K_{1}\right)\right)$ of system (1) is locally asymptotically stable in $\Gamma$ if $\mathscr{R}_{0} \leq 1$ and it is unstable if $\mathscr{R}_{0}>1$.

Proof. We use the Jacobian stability approach to prove the local stability of the morally corrupt-free equilibrium state. Linearization at $E_{0}$ gives the Jacobian matrix as follows:

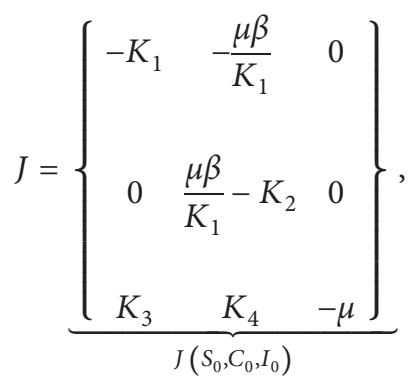

where $J\left(S_{0}, C_{0}, I_{0}\right)$ is the Jacobian matrix of the system of equations (1) evaluated at the equilibrium point $E_{0}$. At each of the equilibrium solutions, we seek solutions to the linear system of the form $\vec{X}=\vec{X}_{0} e^{\lambda t}$, where $\vec{X}=(S, C, I)^{T}, \vec{X}=$ $\left(S_{0}, C_{0}, I_{0}\right)^{T}$ is a nonzero vector of constants, and $\lambda$ is an eigenvalue that measures the growth or decay rate of solutions to the linearized system. This gives rise to the linear
TABLE 2: Baseline values for parameters of system (1).

\begin{tabular}{lcc}
\hline Parameter & Value & Reference \\
\hline$\Lambda$ & $920000 \mathrm{yr}^{-1}$ & {$[7]$} \\
$\beta$ & $0.50 \mathrm{yr}^{-1}$ & Calculated \\
$\mu$ & $(1 / 10) \mathrm{yr}^{-1}$ & Estimated \\
$\gamma$ & 0.08 & Assumed \\
$\theta$ & 1.0 & Assumed \\
$\alpha$ & $0,0.025,0.05,0.10$ & Varied \\
$\sigma$ & $0,0.02,0.05,0.10$ & Varied \\
$\delta$ & 0.01 & Assumed \\
$\Pi$ & 0.50 & Assumed \\
\hline
\end{tabular}

TABle 3: Morally corrupt-basic reproduction number $\mathscr{R}_{0}$ for different values of $\sigma$.

\begin{tabular}{lc}
\hline$\sigma$ & $\mathscr{R}_{0}$ \\
\hline 0 & 2.525 \\
0.02 & 1.923 \\
0.05 & 1.359 \\
0.10 & 0.8503 \\
\hline
\end{tabular}

TABLE 4: Morally corrupt-basic reproduction number $\mathscr{R}_{0}$ for different values of $\alpha$.

\begin{tabular}{lc}
\hline$\alpha$ & $\mathscr{R}_{0}$ \\
\hline 0.025 & 1.8066 \\
0.050 & 1.6103 \\
0.1 & 0.8503 \\
\hline
\end{tabular}

TABLE 5: Morally corrupt-basic reproduction number $\mathscr{R}_{0}$ for different values of $\alpha$ and $\sigma$.

\begin{tabular}{lcc}
\hline$\alpha$ & $\sigma$ & $\mathscr{R}_{0}$ \\
\hline 0 & 0 & 2.5252 \\
0.025 & 0.02 & 1.43369 \\
0.05 & 0.05 & 0.85034 \\
0.10 & 0.10 & 0.42445 \\
\hline
\end{tabular}

system of algebraic equations $(J \lambda I) \overrightarrow{X_{0}}=\overrightarrow{0}$, which has nonzero solutions when

$$
|J-\lambda I|=0 .
$$

The reduced echelon matrix of (7) is

$$
J=\left\{\begin{array}{ccc}
-K_{1} & -\frac{\mu \beta}{K_{1}} & 0 \\
0 & \frac{\mu \beta}{K_{1}}-K_{2} & 0 \\
0 & 0 & -\mu
\end{array}\right\} .
$$

The characteristics polynomial of matrix (9) is 


$$
\left|\left(F V^{-1}\right)-\lambda I_{3}\right|=\left|\begin{array}{ccc}
-K_{1}-\lambda & -\frac{\mu \beta}{K_{1}} & 0 \\
0 & \left(\frac{\mu \beta}{K_{1}}-K_{2}\right)-\lambda & 0 \\
0 & 0 & -\mu-\lambda
\end{array}\right|=0 \text {, }
$$

where $\lambda$ is the eigenvalue of $J_{E_{0}}$. The characteristic equation (10) admits three roots as follows:

$$
\begin{aligned}
& \lambda_{1}=-K_{1}<0, \\
& \lambda_{2}=-\mu<0, \\
& \lambda_{3}=K_{2}\left(\mathscr{R}_{0}-1\right) .
\end{aligned}
$$

Thus, from system (11), $\lambda_{3}<0$ if and only if $\mathscr{R}_{0}<1$. Using the Routh-Hurwitz criterion [22], it can be seen that all the eigenvalues of the characteristic polynomial (10) have negative real part provided $\mathscr{R}_{0}<1$. Hence, $E^{0}$ is LAS on $\Gamma$ provided the inequality $\mathscr{R}_{0}<1$ is satisfied. This completes the proof.

The epidemiological implication of Theorem 1 is that corrupt morals can be effectively controlled (or eliminated) from the population (when $\mathscr{R}_{0} \leq 1$ ) if the initial sizes of the subpopulations of the model are in the region of attraction of the (MCFE) $\left(E_{0}\right)$. To ensure the effective control (or elimination) of corrupt morals from the society is independent of the initial size of the subpopulations of model (1), it is necessary to show that the MCFE is globally asymptotically stable (GAS). This is considered in Theorem 2 that follows immediately.

\subsection{Global Stability of MCFEP}

Theorem 2. The MCFE, $E_{0}$, of model (1), given by (4), is GAS in $\Gamma$ if $\mathscr{R}_{0} \leq 1$.

Proof. Construction of appropriate Lyapunov function is one of the approach used in studying the global asymptotic stability of the MCFE. Consider the constructed Lyapunov function given as follows:

$$
\mathscr{F}=d_{1} C(t),
$$

where $d_{1}=\mu$. Obviously, $\mathscr{F}$ is $C^{1}, \mathscr{F}\left(E^{0}\right)=0$, and $\mathscr{F}$ attains the global minimum 0 in $\Gamma$ only at $E^{0}$. The associated Lyapunov derivative is given by the following (where a dot represents differentiation with respect to time $\mathrm{t}$ ):

$$
\dot{\mathscr{F}}=d_{1} \frac{\mathrm{d} C}{\mathrm{~d} t} .
$$

Substituting in for $(\mathrm{d} C / \mathrm{d} t)$ yields

$$
\dot{\mathscr{F}}=d_{1}\left(\beta \frac{C(t) S(t)}{N(t)}-K_{2} C(t)\right) .
$$

Factoring in $d_{1}$ and simplifying equation (14) result to

$$
\dot{\mathscr{F}}=\mu\left[\beta \frac{S(t)}{N(t)}-K_{2}\right] C(t) .
$$

Since $\left(S_{0} / N_{0}\right) \leq\left(S^{*}(t) / N^{*}(t)\right)$, we have

$$
\begin{aligned}
\dot{\mathscr{F}} & =\mu\left\{\left[\beta \frac{S(t)}{N(t)}-K_{2}\right] C\right\} \\
& \leq\left[\beta \frac{S^{*}(t)}{N^{*}(t)}-K_{2}\right] C .
\end{aligned}
$$

Simplifying equation (16) and factoring in expression (6) reduce to

$$
\dot{\mathscr{F}} \leq\left[\mu K_{2}\left(\mathscr{R}_{0}-1\right)\right] C .
$$

Hence, $\dot{\mathscr{F}} \leq 0$ holds if $\mathscr{R}_{0} \leq 1$. Furthermore, $\dot{\mathscr{F}}=0$ if and only if $C=0$. Let $V=(S, C, I) \in \Gamma \mid \dot{\mathscr{F}}=$ $0=\left(\left(\Lambda / K_{1}\right), 0,\left(K_{3} \Lambda / \mu K_{1}\right)\right)$, then the largest compact invariant set in $\Gamma$ for system (1) is the set $\left(\left(\Lambda / K_{1}\right)\right.$, $\left.0,\left(K_{3} \Lambda / \mu K_{1}\right)\right)$. Thus, the solution of system (1) satisfies $C \longrightarrow 0$ as $t \longrightarrow \infty$ by LaSalle's invariance principle [23]. Therefore, the limit of system (1) is as follows:

$$
\begin{aligned}
& \dot{S}=\frac{\Lambda}{K_{1}}, \\
& \dot{C}=0, \\
& \dot{I}=\frac{K_{3} \Lambda}{\mu K_{1}} .
\end{aligned}
$$

It is obviously known that the equilibrium $E_{0}\left(\left(\Lambda / K_{1}\right), 0,\left(K_{3} \Lambda / \mu K_{1}\right)\right)$ of system (1) is globally asymptotically stable; thus, the morally corrupt-free equilibrium $E_{0}$ is globally attractive in $\Gamma$. On the basis of local stability, $E_{0}$ is globally asymptotically stable in $\Gamma$ if $\mathscr{R}_{0} \leq 1$. This completes the proof.

Theorem 3. This shows that the classical epidemiological requirement of $\mathscr{R}_{0} \leq 1$ is necessary and sufficient for elimination of moral corruption.

3.6. The Endemic Equilibrium Point MCEP Point. In order to establish the existence of morally corrupt endemic equilibria of model (1) (that is, equilibrium where the morally corrupt component of the model is nonzero), the following steps are taken. Let $E^{*}\left[S^{*}, C^{*}, I^{*}\right]$ represent any arbitrary morally corrupt endemic equilibrium of model (1). Solving the equations of model (1) at steady-state gives 


$$
\begin{aligned}
& S^{*} \frac{\Lambda}{\eta^{*}+K_{1}}, \\
& C^{*}=\frac{\eta^{*} \Lambda}{K_{2}\left(\eta^{*}+K_{1}\right)}, \\
& I^{*}=\frac{\eta^{*} \delta \Lambda+K_{2} K_{3} \Lambda}{\mu K_{2}\left(\eta^{*}+K_{1}\right)} .
\end{aligned}
$$

The force of moral corruption $\eta^{*}$ can be expressed, at steady-state, as

$$
\eta^{*}=\beta \frac{C^{*}}{N^{*}}
$$

Substituting the expression for $C^{*}$ and $N^{*}$ where $N^{*}=$ $S^{*}+C^{*}+I^{*}$ in (19) into (20) gives

$$
\eta^{*}=\frac{\mu \beta}{\mu K_{2}+\mu \eta^{*}+K_{2} K_{3}+\delta \eta^{*}} .
$$

It can be easily shown that equation (21) reduces to

$$
\eta^{*}=K_{1}\left(\frac{\mu \beta}{K_{1} K_{2}}-1\right) \text {. }
$$

Hence,

$$
\eta^{*}=K_{1}\left(\mathscr{R}_{0}-1\right),
$$

$\eta^{*}>0$ whenever $\mathscr{R}_{0}>1$ and $\mathscr{R}_{0}<1$ whenever $\eta^{*}<0$. From this, we see that, for $\mathscr{R}_{0}<1$, there is no endemic equilibrium for model (1). Therefore, this condition shows that model (1) exhibits forward bifurcation, if $\mathscr{R}_{0}<1$ (see further proof in subsection 3.7.1). The components of $E^{*}$ can then be obtained by substituting the unique value of $\eta^{*}$ given in (23) into the expressions in (19) to obtain

$$
\begin{aligned}
S^{*} & =\frac{\Lambda}{K_{1} \mathscr{R}_{0}}, \\
C^{*} & =\frac{\Lambda\left(\mathscr{R}_{0}-1\right)}{K_{2} \mathscr{R}_{0}}, \\
I^{*} & =\frac{\delta \Lambda K_{1}\left(\mathscr{R}_{0}-1\right)+K_{2} K_{3} \Lambda}{\mu K_{1} K_{2} \mathscr{R}_{0}} .
\end{aligned}
$$
$\mathscr{R}_{0}>1$.

The endemic equilibrium solution $E^{*}$ exists whenever

Remark 1. From the equilibrium values given by equation (24), when $\mathscr{R}_{0}=1$, the endemic equilibrium $E^{*}$ reduces to the Morally corrupt-free equilibrium $E_{0}$. On the other hand, when $\mathscr{R}_{0} \leq 1$, the only realistic equilibrium solution is $E_{0}$. Hence, the following result is established.

Lemma 2. Model (1) has a unique morally corrupt endemic (positive) equilibrium, given by $E^{*}$, whenever $\mathscr{R}_{0}>1$ and no endemic equilibrium otherwise. We end this section by proving the local as well as global stability of the endemic equilibrium $E^{*}$. Our proof is based on the center manifold theory [24], as described in Theorem 4.1 of [25] and construction of appropriate Lyapunov function.

\subsection{Local Stability of Endemic Equilibrium}

3.7.1. Bifurcation Analysis. Here, the center manifold [24], as described in Theorem 4.1 of [25], will be used to obtain the local asymptotic stability of endemic equilibrium. To use the center manifold theory, we consider a change of variables. Let

$$
Y=\left\{x_{1}, x_{2}, x_{3}\right\}=\{S, C, I\} .
$$

The total number of individuals is thus given by $N=\sum_{1}^{3} x_{i}$, Thus, model (1) can be written as follows:

$$
\begin{aligned}
& \frac{\mathrm{d} x_{1}}{\mathrm{~d} t}=f_{1}=\Lambda-\frac{\beta x_{1} x_{2}}{N}-K_{1} x_{1}, \\
& \frac{\mathrm{d} x_{2}}{\mathrm{~d} t}=f_{2}=\frac{\beta x_{1} x_{2}}{N}-K_{2} x_{2}, \\
& \frac{\mathrm{d} x_{3}}{\mathrm{~d} t}=f_{3}=K_{3} x_{1}+K_{4} x_{2}-\mu x_{3} .
\end{aligned}
$$

Choosing $\beta^{*}$ as bifurcation parameter and solving for $\beta$ from equation (6) by letting $\mathscr{R}_{0}=1$, we obtain

$$
\beta^{*}=\frac{K_{1} K_{2}}{\mu} \text {. }
$$

Considering $\beta=\beta^{*}$, the Jacobian of system (1) evaluated at $E_{0}$ is given by

$$
\left|J^{*}\left(E_{o}\right)-\lambda I\right|=\left|\begin{array}{ccc}
-K_{1}-\lambda & -K_{2} & 0 \\
0 & 0-\lambda & 0 \\
K_{3} & K_{4} & -\mu-\lambda
\end{array}\right|=0 .
$$

The eigenvalues of $J_{0}^{*}$ matrix (28) are

$$
\begin{aligned}
& \lambda_{1}=-K_{1}, \\
& \lambda_{2}=0, \\
& \lambda_{3}=-\mu .
\end{aligned}
$$

We conclude that zero is a simple eigenvalue of $J^{*}\left(E_{0}\right)$, and all other eigenvalues of $J^{*}\left(E_{0}\right)$ have negative real parts. Therefore, the center manifold theory [4] can be applied to study the dynamics of (26) near $\beta=\beta^{*}$. Theorem 4.1 in [25] is used to show the local asymptotic stability of the endemic equilibrium point of (26), for $\beta$ near $\beta^{*}$. The Jacobian $J^{*}\left(E_{0}\right)$ has, respectively, a right eigenvector and a left eigenvector (associated with the zero eigenvalue) $w=\left[w_{1}, w_{2}, w_{3}\right]^{T}$ and $v=\left[v_{1}, v_{2}, v_{3}\right]^{T}$ given by

$$
\begin{aligned}
& w=\left[\frac{-K_{2}}{K_{1}}, 1, \frac{-K_{2} K_{3}+K_{1} K_{4}}{\mu K_{1}}\right]^{T} w_{2}, \\
& v=[0,1,0]^{T} v_{2} .
\end{aligned}
$$


Since, $v_{1}=0$ and $v_{3}=0$, we only need to calculate the partial derivative of $f_{2}$. For system (26), the associated nonzero partial derivative of $f_{2}$ is given by

$$
\frac{\partial^{2} f_{2}}{\partial x_{2} \partial x_{2}}=-\frac{2 \beta}{x_{1}} \text {. }
$$

The local stability near the bifurcation point $\beta=\beta^{*}$ is determined by the signs of two associated constants $a$ and $b$ defined by

$$
\begin{aligned}
a & =\sum_{i, j, \beta=1}^{4} v_{k} w_{i} w_{j}\left(\frac{\partial^{2} f_{\beta}}{\partial x_{i} \partial x_{j}}\left(E_{0}\right)\right)_{\beta=\beta^{*}}, \\
& =v_{2} \sum_{i, j=1}^{3} w_{i} w_{j} \frac{\partial^{2} f_{2}}{\partial x_{i} \partial x_{j}}, \\
& =v_{2} w_{1}^{2} \frac{\partial^{2} f_{2}}{\partial x_{2} \partial x_{2}}, \\
& =-\frac{2 \beta}{x_{1}}, \\
& =-\frac{2 \beta}{\Lambda}\left(\mu+K_{3}\right)<0, \\
b & =\sum_{i, j, \beta=1}^{3} v_{\beta} w_{i}\left(\frac{\partial^{2} f_{\beta}}{\partial x_{i} \partial \beta}\left(E_{0}\right)\right)_{\beta=\beta^{*}} .
\end{aligned}
$$

To determine $b$, we calculate the second-order partial derivative so that

$$
\frac{\partial^{2} f_{2}}{\partial x_{2} \partial \beta^{*}}=1
$$

Therefore, $b$ is obtained as follows:

$$
b=\sum_{i=1}^{3} v_{2} w_{i}\left(\frac{\partial^{2} f_{2}}{\partial x_{i} \partial \beta^{*}}\right)_{\beta=\beta^{*}} .
$$

Expression (35) reduces to

$$
b=v_{2} \cdot w_{2} \cdot(1)
$$

where $v_{2}$ and $w_{2}$ are as in equations (30) and (31), respectively; therefore;

$$
b=1>0 .
$$

Thus, $a<0, b>0$. Therefore, by item (iv) of Theorem 4.1 in [25], we can write the following result.

Theorem 4. The unique endemic equilibrium is locally asymptotically stable for $\mathscr{R}_{0}$ near 1 , (that is, the result in Theorem 4.1 in [25] holds for $\mathscr{R}_{0}>1$ but close to 1). In other words, model (1) has a global asymptotically stable MCFE whenever $\mathscr{R}_{0} \leq 1$ and a unique endemic equilibrium whenever $\mathscr{R}_{0}>1$.

\subsection{The Global Stability of Endemic Equilibrium Point}

Theorem 5. If $\mathscr{R}_{0}>1$, then the unique endemic equilibrium $E^{*}$ of (1) is globally asymptotically stable in $\Gamma$. In the following proof, we use LaSalle's invariance principle to establish the globally asymptotic stability of $E^{*}$. We need a result on persistence of (1), before proceeding to proof of global asymptotic stability of $E^{*}$. It is easy to see that a solution $S(t)$, $C(t)$, and $I(t)$ of (1) with $S(0)>0+C(t)>0+I(t)>0$ is eventually (component-wise) positive. Hence, for the global stability of the endemic equilibrium $E^{*}$, we only need to consider the interior of the feasible region $\Gamma$ :

$$
\left.\Gamma^{0}=(S, C, I \in \Gamma: S>0, C>0, I>0)\right),
$$

since it is also a positively invariant set of (1). This is achieved with an algebraic approach as in [26] to construct a suitable Lyapunov function. With similar approaches as those in [27], it can easily be proven that system (1) is uniformly persistence.

Definition 3 (see $[28,29]$ ). System (1) is said to be uniformly persistent if there exists a constant $n \in(0,1)$ such that any solution $(\mathrm{S}(\mathrm{t}), \quad \mathrm{C}(\mathrm{t}), \quad \mathrm{I}(\mathrm{t}))$ with initial point $(S(0), C(0), I(0)) \in \Gamma^{0}$ satisfies

$$
\left[\lim _{t \longrightarrow \infty} \inf S(t), \lim _{t \longrightarrow \infty} \inf C(t), \lim _{t \longrightarrow \infty} \inf I(t)\right]>n .
$$

Lemma 3. When $\mathscr{R}_{0}>1$, system (1) is uniformly persistent in $\Gamma^{0}$ (see [30] for more proof).

This, combined with Lemma 3, implies that (1) is uniformly persistent if $\mathscr{R}_{0}>1$. Therefore, system (1) has a compact absorbing set $G \subset \Gamma$ (see, for example, [26]).

Proof. We only need to prove that $E^{*}$ is globally asymptotically stable in a compact absorbing set of $\Gamma$, which exists as we have argued above. Let $G$ be such a compact absorbing set. Define a Lyapunov function $L: G \longrightarrow \mathscr{R}_{0}$.

$$
L(S, C)=x_{1}\left(S-S^{*} \ln \frac{S}{S^{*}}\right)+x_{2}\left(C-C^{*} \ln \frac{C}{C^{*}}\right),
$$

where $x_{1}>0$ and $x_{2}>0$ are positive constants to be chosen later. It is clear that $L$ is $C^{1}, L\left(E^{*}=0\right)$, and $L$ is strictly positive at other points in G. The time derivative of $L$ along the solutions of (1) is given by

$$
\frac{\mathrm{d} L}{\mathrm{~d} t}=x_{1}\left(1-\frac{S^{*}}{S}\right) \frac{\mathrm{d} S}{\mathrm{~d} t}+x_{2}\left(1-\frac{C^{*}}{C}\right) \frac{\mathrm{d} C}{\mathrm{~d} t} .
$$

Substituting in for $(\mathrm{d} S / \mathrm{d} t)$ and $(\mathrm{d} C / \mathrm{d} t)$ from system of equations (1) in equation (41), we have

$$
\begin{aligned}
\left.\frac{\mathrm{d} L}{\mathrm{~d} t}\right|_{(1)}= & x_{1}\left(1-\frac{S^{*}}{S}\right)\left(\Lambda-\frac{\beta C S}{N}-K_{1} S\right) \\
& +x_{2}\left(1-\frac{C^{*}}{C}\right),\left(\frac{\beta C S}{N}-K_{2} C(t)\right),
\end{aligned}
$$

and by expanding equation (42), we get 


$$
\begin{aligned}
\left.\frac{\mathrm{d} L}{\mathrm{~d} t}\right|_{1}= & x_{1} \Lambda-x_{1} \frac{\beta C S}{N}-x_{1} K_{1}\left(S-S^{*}\right)-x_{1} \Lambda \frac{S^{*}}{S} \\
& +x_{1} \frac{\beta C S^{*}}{N},+x_{2} \frac{\beta C S^{*}}{N}-x_{2} K_{2}\left(C-C^{*}\right)-x_{2} \frac{\beta C^{*} S}{N} .
\end{aligned}
$$

From system of equations (1), we have

$$
\begin{aligned}
& K_{1}=\frac{\Lambda}{S^{*}}-\frac{\beta C^{*}}{N}, \\
& K_{2}=\frac{\beta S^{*}}{N} .
\end{aligned}
$$

Then,

$$
\begin{aligned}
\left.\frac{\mathrm{d} L}{\mathrm{~d} t}\right|_{1}= & x_{2} \frac{\beta C S}{N}-x_{2} \frac{\beta S C^{*}}{N}-x_{1} \frac{\beta C S}{N}+x_{1} \frac{\beta C^{*} S}{N}-x_{2} \frac{\beta C S^{*}}{N} \\
& +x_{2} \frac{\beta C^{*} S^{*}}{N}+x_{1} \frac{\beta C S^{*}}{N}-x_{1} \frac{\beta C^{*} S^{*}}{N}-x_{1} \Lambda\left(\frac{S}{S^{*}}-2+\frac{S^{*}}{S}\right) .
\end{aligned}
$$

Factorizing equation (45), we have

$$
\frac{\mathrm{d} L}{\mathrm{~d} t}=\frac{\beta}{N}\left(x_{2}-x_{1}\right)\left(S-S^{*}\right)\left(C-C^{*}\right)-\Lambda x_{1} \frac{\left(S-S^{*}\right)^{2}}{S S^{*}} .
$$

For $x_{1}=x_{2}=1$, we have

$$
\frac{\mathrm{d} L}{\mathrm{~d} t}=-\Lambda \frac{\left(S-S^{*}\right)^{2}}{S S^{*}} \leq 0 .
$$

Thus, $\dot{L}(S, C) \leq 0$ only if $S=S^{*}$. Therefore, the largest invariant set of system of equations in (1) on the set $\left\{(S, C, I) \in \Gamma_{+}^{3}: \dot{L}(S, C, I)=0\right\}$ is the endemic equilibrium point $E^{*}$. Thus, by LaSalle's invariance principle [23], it follows then that the endemic equilibrium $E^{*}$ is globally asymptotically stable. This concludes the proof.

3.9. Sensitivity Analysis. Sensitivity analysis helps us to know the parameters that have a great impact on the reproduction number $\mathscr{R}_{0}$ so that efforts to curb the problem are directed to these parameters. We perform sensitivity analysis as defined in [31]. Sensitivity analysis of model (1) is carried out in a similar way to [32] where the definition of normalized forward sensitivity index defined as a variable, $\mathscr{R}_{0}$, that depends differentiably on a parameter, $\mathscr{L}$, is defined as follows:

$$
\sqcup_{\mathscr{L}}^{\mathscr{R}_{0}}=\frac{\partial \mathscr{R}_{0}}{\partial \mathscr{L}} \cdot \frac{\mathscr{L}}{\mathscr{R}_{0}},
$$

for $\mathscr{L}$ represents all the basic parameters and $\mathscr{R}_{0}$ is as in equation (6).

$$
\left\{\begin{array}{l}
\sqcup_{\beta}^{\mathscr{R}_{0}}=\frac{\partial \mathscr{R}_{0}}{\partial \beta} \cdot \frac{\beta}{\mathscr{R}_{0}}=1 \geq 0, \\
\sqcup_{\delta}^{\mathscr{R}_{0}}=\frac{\partial \mathscr{R}_{0}}{\partial \delta} \cdot \frac{\delta}{\mathscr{R}_{0}}=-\frac{\delta}{\delta+\alpha+\sigma+\mu} \leq 0, \\
\sqcup_{\gamma}^{\mathscr{R}_{0}}=\frac{\partial \mathscr{R}_{0}}{\partial \gamma} \cdot \frac{\gamma}{\mathscr{R}_{0}}=-\frac{\gamma}{\gamma+\alpha+\sigma+\mu} \leq 0 .
\end{array}\right.
$$

The remaining parameters are done in a similar way, and sensitivity indices are as found in Table 6 .

3.10. Interpretation of Sensitivity Indices. The parameter that have positive index such as $\Pi$ and $\theta$ shows that they have a high impact on ballooning the moral corruption in the society if their values are increasing. Furthermore, those parameters with negative sensitivity indices $\delta, \alpha, \gamma, \sigma$, and $\mu$ minimize the burden of moral corruption in the society as their value increases while the others are left unchanged. Increasing these parameters with negative indices corresponds to reduction in basic reproduction number $\mathscr{R}_{0}$, which leads to minimizing the endemicity of the corruption of morals in the society. Thus, the model sensitivity analysis bespoke that policymakers are supposed to diminish positive index parameters and increase negative index parameters in order to combat moral corruption in the society. With sensitivity analysis, one can get insight into the appropriate intervention strategies to prevent and control the spread of teenage morals corruption as described by model (1).

\section{Numerical Simulations}

4.1. Demographic Parameters. Numerical simulations of model (1) are presented based on demographic data relevant to adolescent population of Kenya as follows. According to [7], an adolescent is any person aged between 10 and 19 years, and the average stay in this bracket is estimated as 10 years. This study, therefore, assumes constant exit rate $\mu$ to be the reciprocal of the average stay in this bracket. Thus, $\mu=0.1$ and recruitment rate $\Lambda$ is assumed to be $\Lambda=920000$ per year, which is used for numerical simulations, so that $N=(\Lambda / \mu)=9,200,000$, which is the current estimated adolescents population in Kenya as per [7,33]. In addition, the following initial conditions will be used in simulations of model (1): $\quad S(0)=4,200,000, \quad C(0)=3,000,000$, and $I(0)=2,000,000$, so that the total initial morally corrupt population is $3,000,000$. In other words, for the purposes of numerical simulations, the high estimate of $3,000,000$ morally corrupt population is considered to account for any uncertainty in the estimate for the current total number of morally corrupt population in Kenya. The parameter values for simulation are provided in Table 2 .

Using the aforementioned set of demographic data and the associated epidemiological data relevant to Kenyan adolescent population given in Table 2, model (1) gives a 
TABle 6: Sensitivity index table.

\begin{tabular}{lc}
\hline Parameter symbol & Sensitivity indices \\
\hline$\beta$ & $+\mathrm{ve}$ \\
$\Pi$ & $+\mathrm{ve}$ \\
$\theta$ & $+\mathrm{ve}$ \\
$\alpha$ & $-\mathrm{ve}$ \\
$\sigma$ & $-\mathrm{ve}$ \\
$\delta$ & $-\mathrm{ve}$ \\
$\gamma$ & $-\mathrm{ve}$ \\
$\mu$ & $-\mathrm{ve}$ \\
\hline
\end{tabular}

practical fit of the observed morally corrupt population for Kenya. This suggests that the model can be used to gain practical insight into transmission of moral corruption amongst the adolescents in Kenya and to evaluate various moral corruption control strategies.

4.2. Assessment of Control Strategies. This study considers two main corrupt moral control strategies, namely:

(i) Comprehensive training on life skills, provision of age-appropriate sexual information, and availability of youth friendly services in place

(ii) Guidance and counselling process

Notice that in the absence of any intervention, that is setting $\alpha=\sigma=0$, the reproduction number $\mathscr{R}_{0}$ of model (1) reduces to

$$
\mathscr{R}_{0}=\frac{\mu \beta}{(\gamma+\mu)(\delta+\mu)} .
$$

Using the data from Table 2, the reproduction number $\mathscr{R}_{0}$ corresponding to equation (50) is $\mathscr{R}_{0}=2.5$, that is, over half of the adolescent population will be morally corrupted in the span of 5 years if no interventions is employed (see Figure 2).

In the subsequent section, we will assess the two interventions separately as well as when combined.

\subsection{Assessment of Single Interventions}

4.3.1. Comprehensive Training on Life Skills, Provision of Age Appropriate Sexual Information, and Availability of Youth Friendly Services in Place. Here, we assess the impact of comprehensive training on life skills, provision of age-appropriate sexual information, and availability of youth friendly services. Essential commodities that should be availed on the adolescent and youth friendly service delivery points are accessible in terms of privacy, cost, and distance but not limited to supplies and medicines for sexually transmitted infections management, pregnancy testing kits, contraceptives, HIV testing services kits, condoms, postabortal care kits, sanitary pads, antiretroviral drugs, post rape care kits, and so on. Here, for purpose of simulation, we set $\alpha=0$. The assumption here is that if the age-appropriate sexual information is readily available and youth friendly services are equitable, accessible, acceptable, appropriate, effective, and available, then $0<\sigma<1$. Letting all the model parameters related to counselling to zero, that is, $\alpha=0$ gives a reduced model with

$$
\mathscr{R}_{0}=\frac{\mu \beta}{(\gamma+\sigma+\mu)(\delta+\sigma+\mu)} .
$$

Furthermore, by differentiating the expression for $\mathscr{R}_{0}$ partially with respect to $\sigma$ gives

$$
\frac{\partial \mathscr{R}_{0}}{\partial \sigma}=\frac{-\mu \beta(\gamma+\delta+2 \alpha+2 \mu)}{(\gamma+\sigma+\mu)^{2}(\delta+\sigma+\mu)^{2}} .
$$

Thus, $\mathscr{R}_{0}$ is a decreasing function of $\sigma$ (see Table 3 ), and Figure 3 shows a decrease in $\mathscr{R}_{0}$ with increasing comprehensive training on life skills, provision of age-appropriate sexual information, and availability of youth friendly services in place, $\sigma$.

The analysis above shows that even a small change in provision of life skills and age-appropriate sexual information $\sigma$ to susceptible and morally corrupt adolescents results in reduction of the reproduction number $\mathscr{R}_{0}$ signifying a reduction in societal moral corruption thereby reducing adolescents moral corruption and pregnancy burden.

To achieve this, a consistence provision and availability of these youth friendly services, comprehensive training on life skills, and provision of age-appropriate sexual information are required. Relying on this strategy alone may not yield expected results, considering the fact that at the moment, the coverage of adolescent youth friendly services (2005) has remained unacceptably low at $\alpha=0.07$ and $\sigma=$ 0.02 which has led to poor adolescent and youth friendly sexual reproductive health services indicators [7]. Furthermore, high level of $\sigma$ guarantees the reduction of $\mathscr{R}_{0}$ to less than a unit. In summary, this study shows that the prospect of effective controlling (or even eliminating) of corruption of morals in Kenya is reasonably high if some basic and affordable steps are taken. This clearly requires significant attention, resources, determination, and willingness on the part of the Kenyan government and all other relevant stake holders.

4.3.2. Guidance Counselling Process Only. To study the impact of counselling process as a single control strategy to moral corruption, we set $\sigma=0$. Thus, $\mathscr{R}_{0}$ of the reduced model (1) is

$$
\mathscr{R}_{0}=\frac{\mu \beta}{(\gamma+\alpha+\mu)(\delta+\alpha+\mu)},
$$

$\alpha$ decreases the value of $\mathscr{R}_{0}$ which is an indication that $\mathscr{R}_{0}$ is a decreasing function of $\alpha$ (see Table 4 and Figure 4$)$. However, relying on counselling process as a single strategy to address sexuality issues in Kenya is not promising. Partial derivative of $\mathscr{R}_{0}$ with counselling rate $\sigma$ yields 


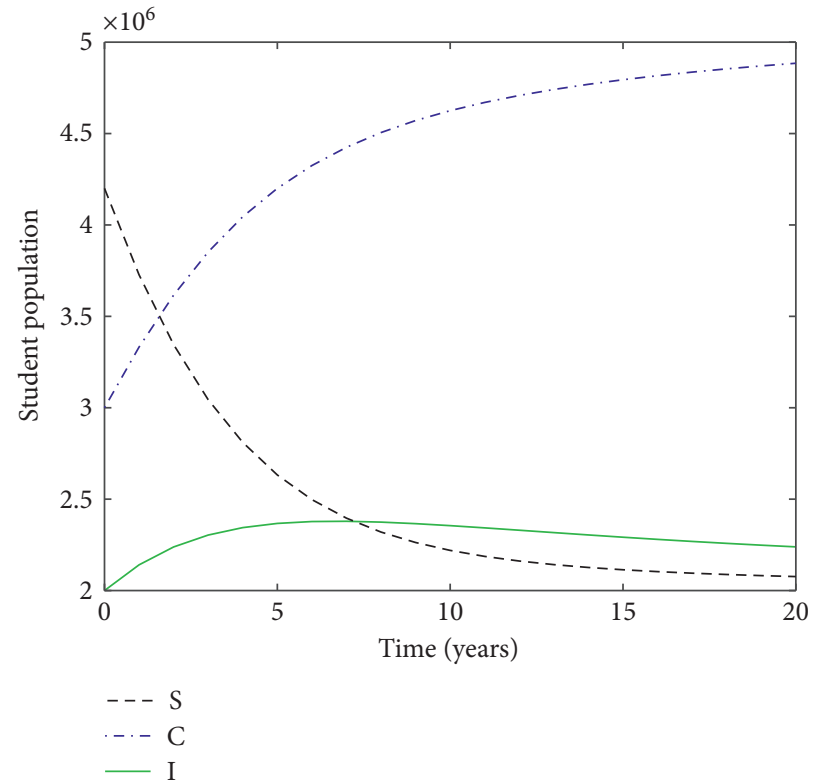

(a)

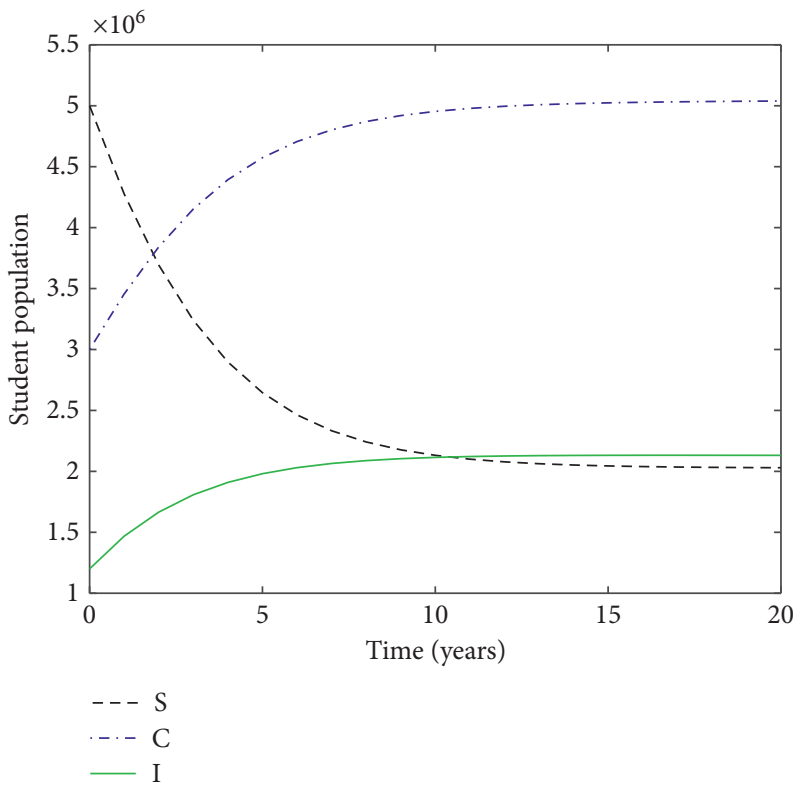

(b)

FIGURE 2: Simulation of the model showing the total number of Susceptible, Morally corrupt, and Immune as a function of time using initial conditions given in Section 2. Parameters used are in Table 2 with $\sigma=0, \alpha=0$, and $\mathscr{R}_{0}=2.525$.

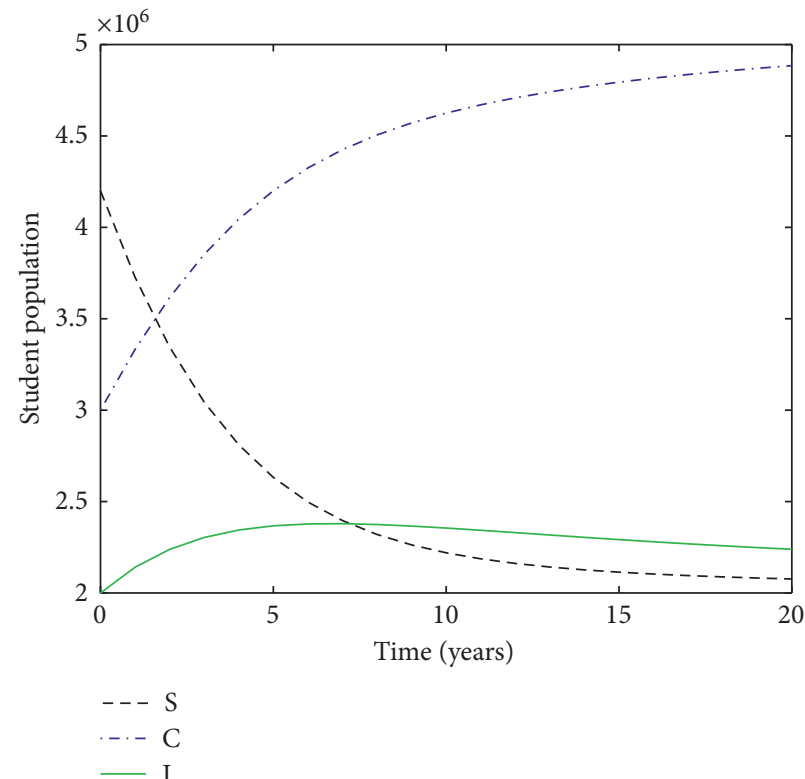

(a)

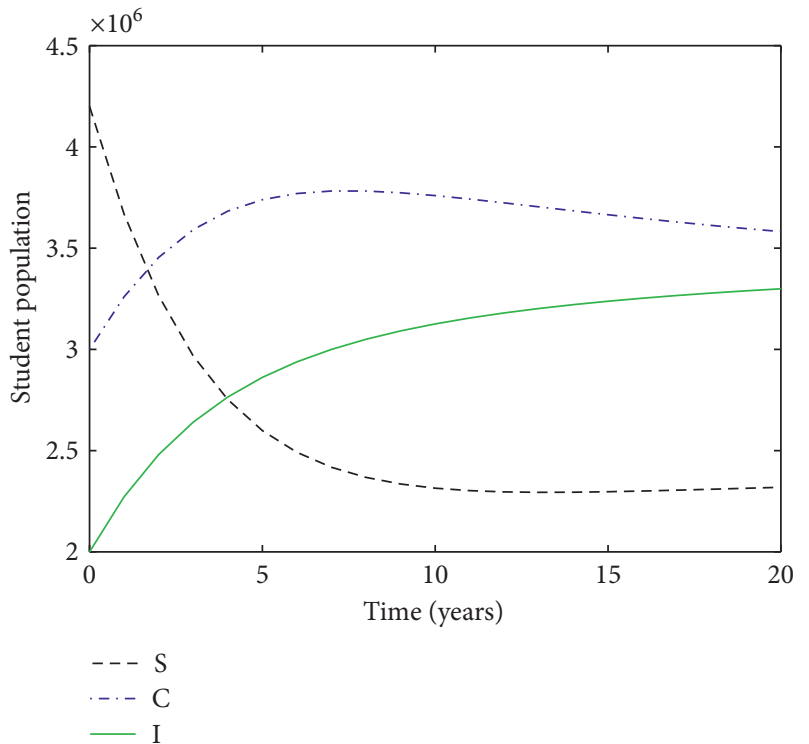

(b)

Figure 3: Continued. 


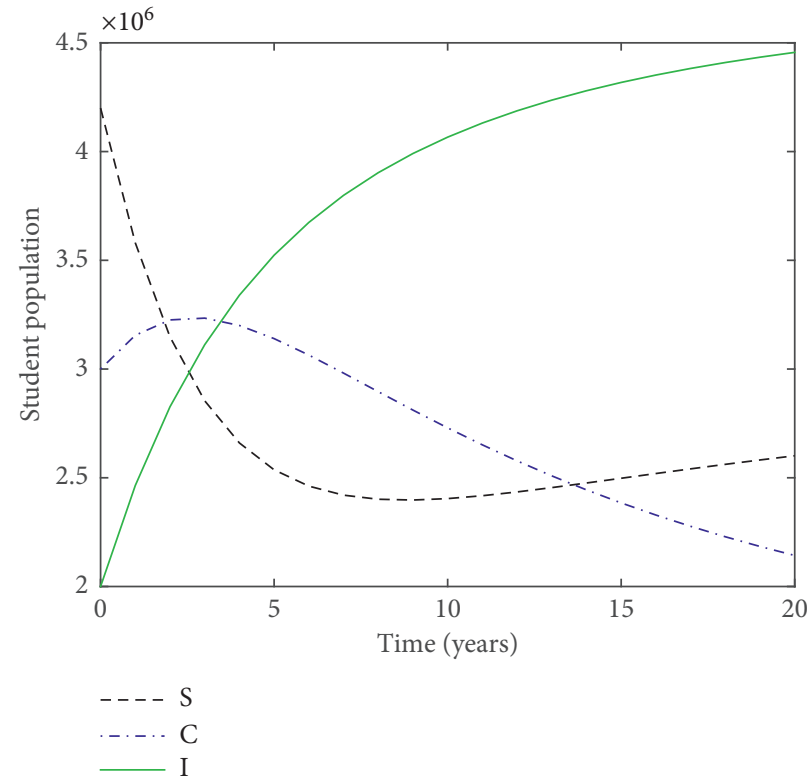

(c)

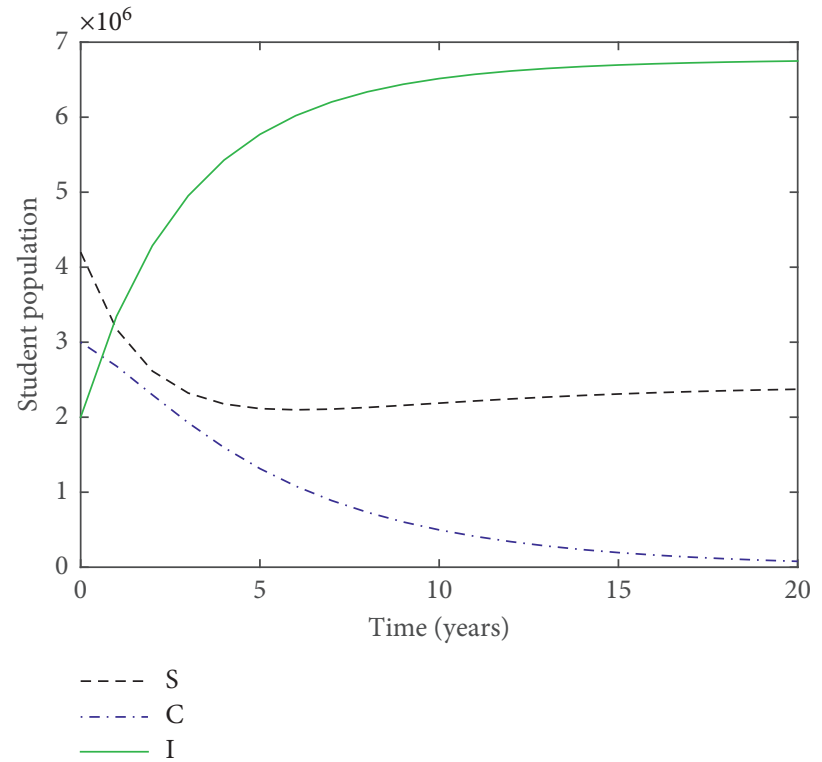

(d)

Figure 3: Simulation of the model showing the total number of Susceptible, Morally corrupt, and Immune as a function of time using initial conditions given in Section 2. Parameters used are in Table 2 with (a) $\sigma=0, \alpha=0, \mathscr{R}_{0}=2.525$; (b) $\sigma=0.02, \alpha=0, \mathscr{R}_{0}=1.923$; (c) $\sigma=0.05$, $\alpha=0, \mathscr{R}_{0}=1.359$; (d) $\sigma=0.20, \alpha=0 . \mathscr{R}_{0}=0.8503$.

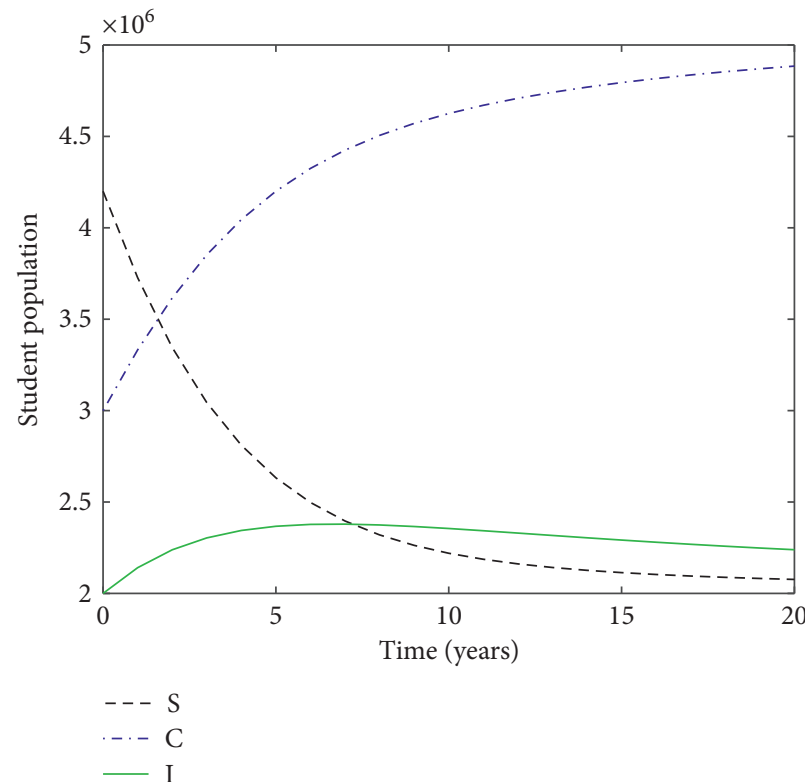

(a)

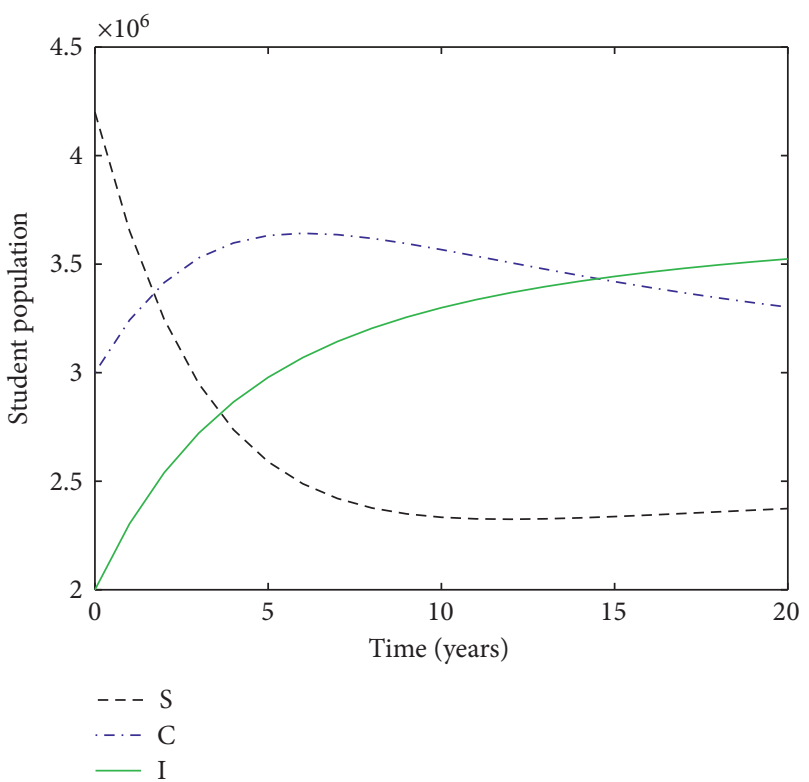

(b)

FIgURe 4: Continued. 


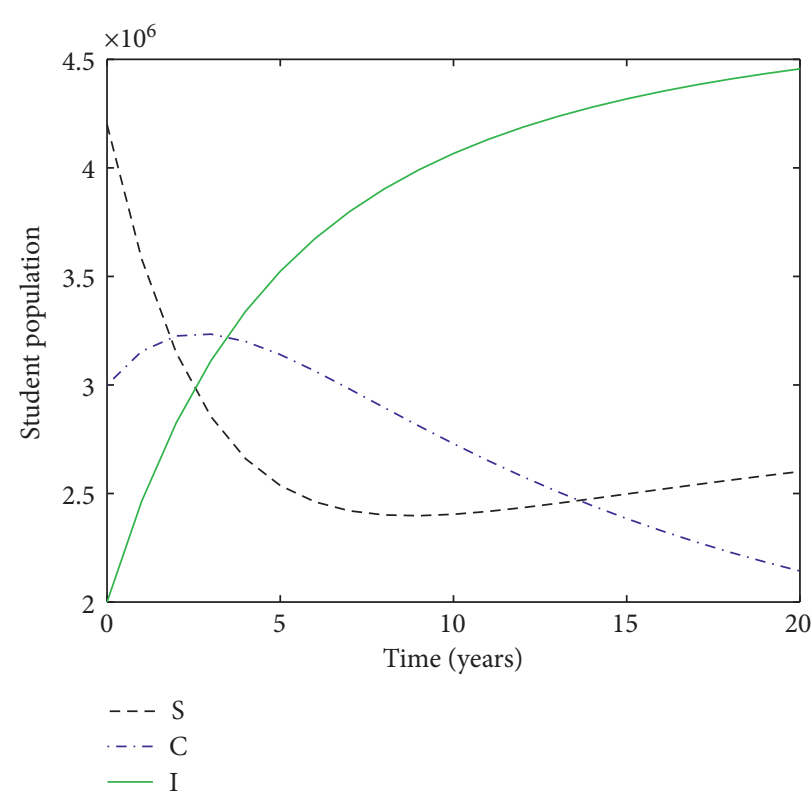

(c)

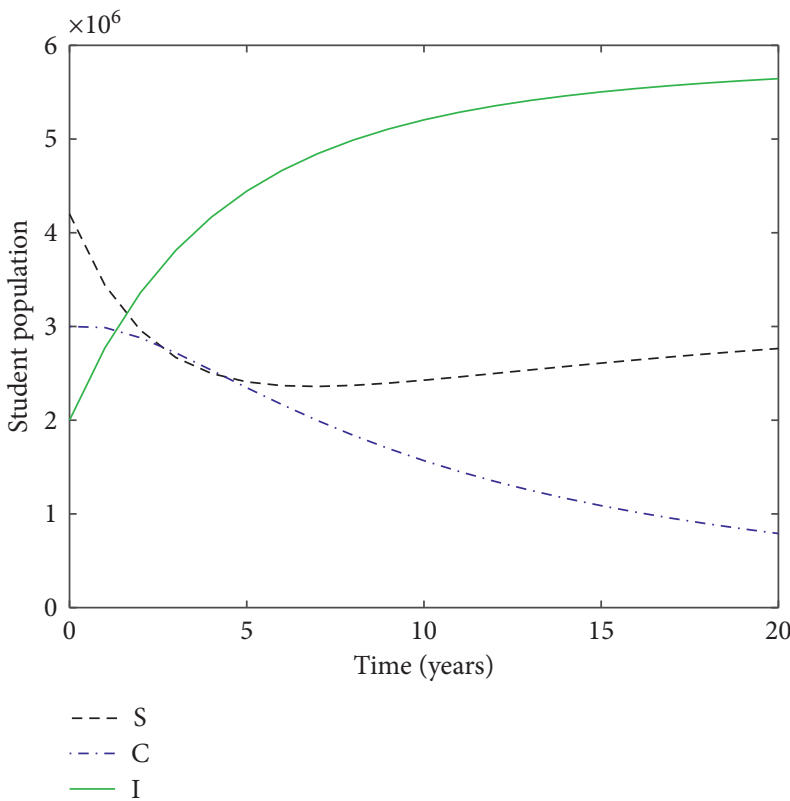

(d)

FIGURE 4: Simulation of the model showing the total number of Susceptible, Morally corrupt, and Immune as a function of time using initial conditions given in Section 2. Parameters used are in Table 2 with (a) $\sigma=0, \alpha=0, \mathscr{R}_{0}=2.525$; (b) $\sigma=0, \alpha=0.025, \mathscr{R}_{0}=1.923$; (c) $\sigma=0$, $\alpha=0.05, \mathscr{R}_{0}=1.359 ;$ (d) $\sigma=0, \alpha=0.10, \mathscr{R}_{0}=0.8503$.

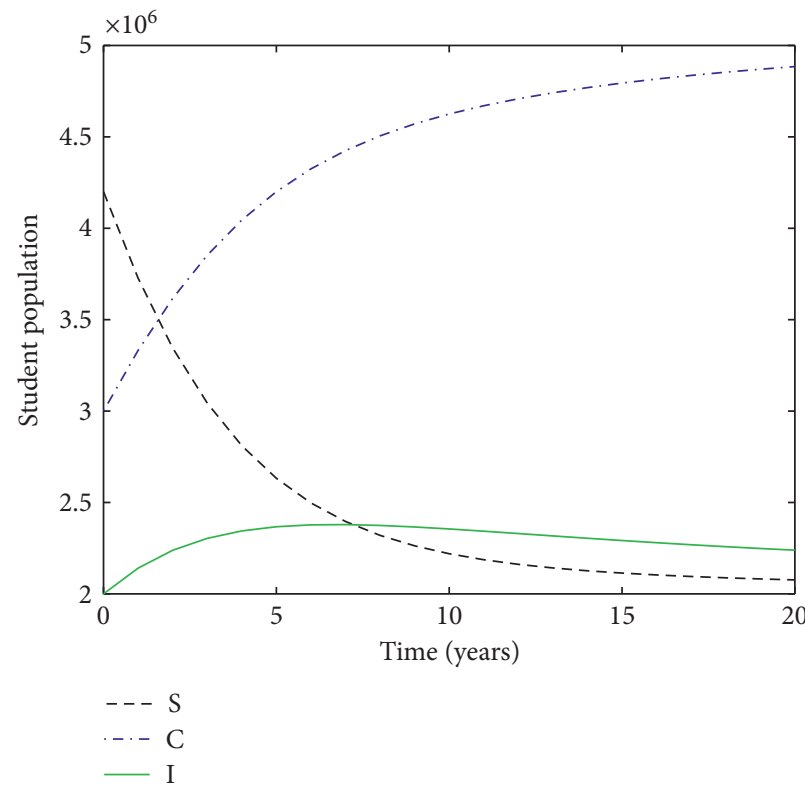

(a)

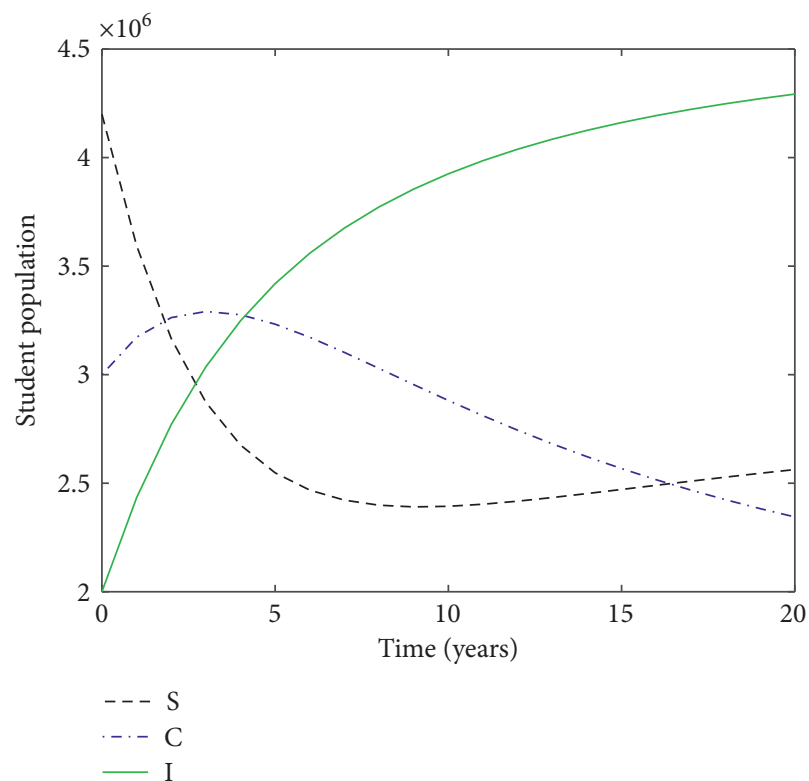

(b)

Figure 5: Continued. 


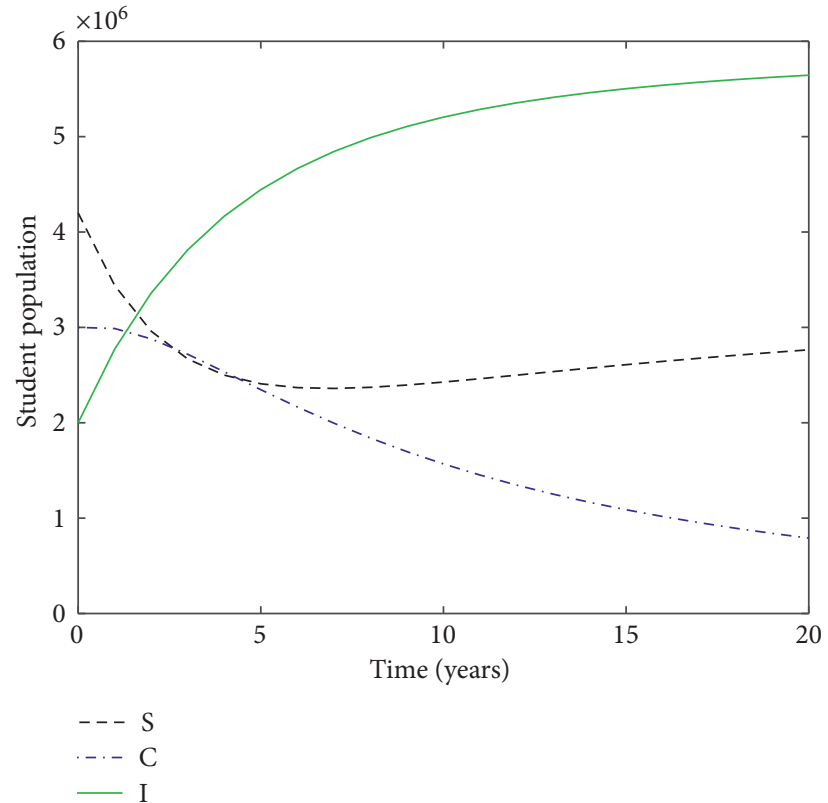

(c)

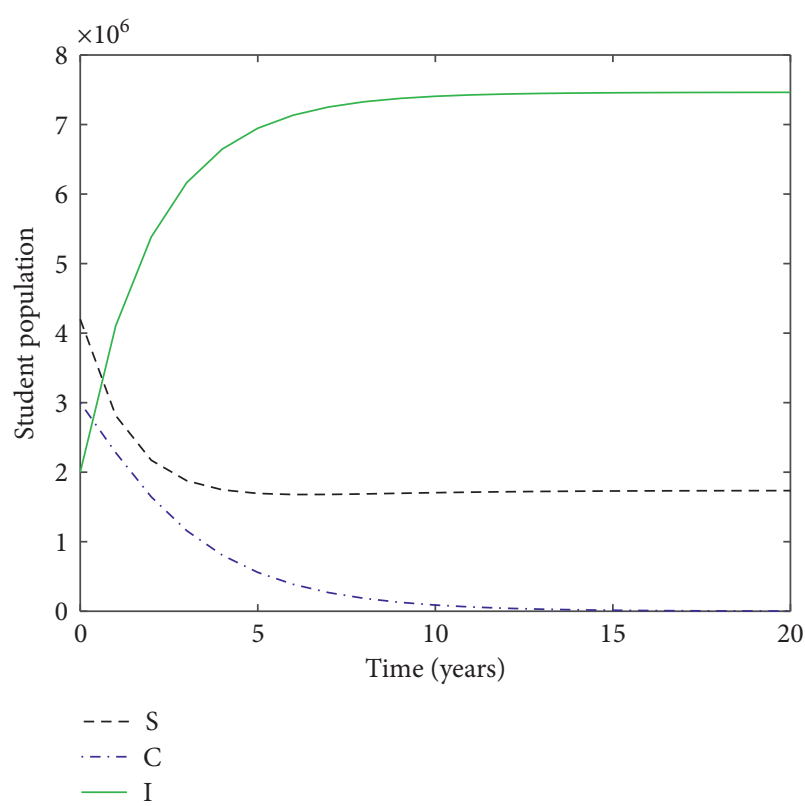

(d)

Figure 5: Simulation of the model showing the total number of Susceptible, Morally corrupt, and Immune as a function of time using initial conditions given in Section 2. Parameters used are in Table 2 with (a) $\sigma=0, \alpha=0, \mathscr{R}_{0}=2.525$; (b) $\sigma=0.02, \alpha=0.025, \mathscr{R}_{0}=1.923$; (c) $\sigma=0.05, \alpha=0.05, \mathscr{R}_{0}=1.359$; (d) $\sigma=0.20, \alpha=0.15, \mathscr{R}_{0}=0.8503$.

$$
\frac{\partial \mathscr{R}_{0}}{\partial \sigma}=-\frac{\mu \beta(\gamma+\delta+2 \alpha+2 \mu)}{(\gamma+\alpha+\mu)^{2}(\delta+\alpha+\mu)^{2}}
$$

which is inversely proportional to $\mathscr{R}_{0}$.

\subsection{Combined Interventions}

4.4.1. Universal Strategy. Further simulations are carried out to monitor the impact of the universal strategy where the two control strategies are incorporated in combination to combat the problem under study. Here, all parameters and variables are nonzero; in this case, $\mathscr{R}_{0}$ is

$$
\mathscr{R}_{0}=\frac{\mu \beta}{(\gamma+\alpha+\sigma+\mu)(\delta+\alpha+\sigma+\mu)},
$$

with different parameter values from Table 2 yield Table 5

Observe from Table 5 and Figure 5 that with approximately 20 percent provision of youth friendly services, information on age-appropriate sexual information and life skills training and counselling level of about 15 percent will probably bring $\mathscr{R}_{0}$ to less than a unit in a span of 10 years if done in adherence and consistency (see Figure 5(d)). Notice that with a combination of the two strategies, $\mathscr{R}_{0}$ is reduced to less than a unity with minimal effort and cost. This model prediction is consistent with available literature [7] that for effective provision of AYFS, it requires a multisectoral approach and coordination for effective control of teen and youth moral corruption.

Numerical simulations of the model, using appropriate demographic and epidemiological data for Kenya, indicate the following:
(1) The use of comprehensive training on life skills, provision of age-appropriate sexual information, and availability of youth friendly services as a sole anticorruption of moral strategy can lead to the effective control of corruption of morals in Kenya if it is equitable, accessible, acceptable, appropriate, and effective in its provision (with at least 0.1 or 10 percent provision of this services). It is, however, unclear if these high levels can be realistically attained in Kenya. Similarly, the singular use of counselling of morally corrupt and susceptible adolescents (to positively modify their risky sexual behaviour) can lead to effective control and/or elimination of corruption of morals in Kenya.

(2) The combined use of comprehensive training on life skills, provision of age-appropriate sexual information, and availability of youth friendly services and counselling (that is, the universal strategy), even if implemented with a low effectiveness level, can lead to corrupt morals elimination in Kenya. The time required to decrease or eliminate the corrupt morals decreases with increasing effectiveness level of the universal strategy.

\section{Conclusion}

In this paper, we derived and analysed a deterministic model for the spread of corrupt morals that includes the class of individuals undergoing guidance and counselling process. We calculated the basic reproduction number $\mathscr{R}_{0}$, investigated the 
existence and stability of equilibria, and performed numerical simulations to ascertain the theoretical (results) analysis. Through Lasselle's invariance principle of Lyapunov functions, the morally corrupt-free and the endemic equilibrium points were proven to be globally asymptotically stable whenever $\mathscr{R}_{0}<1$ and $\mathscr{R}_{0}>1$, respectively. When $\mathscr{R}_{0}=1$, bifurcation analysis of the model was proven and it exhibits forward bifurcation. From our numerical results, we found that the classical epidemiological requirement of $\mathscr{R}_{0} \leq 1$ is necessary and sufficient for elimination of moral corruption vice in the society. Lastly, from sensitivity analysis, it can be inferred that positive indices should be decreased (by providing the adolescents with necessary and sufficient skills and information required to navigate through the already polluted and morally corrupt society and by controlling harmful contents accessed by the young population) while increasing negative indices (by provision of youth friendly services, age-appropriate sexual information, necessary life skills, and guidance and counselling both at places of worship, home, and school levels) to minimize/control moral corruption in the population. Designing optimal control strategies is left for future work.

5.1. Recommendation. Talking and training of online safety is a key mediation strategy. If the Government through Kenya Film Classification Board (KFCB) cannot control the content accessed online to our dear children, then it wise to have clear training on the Internet safety tools such as network-level content filters, parental control software content filters, parental controls built into device, PINs/ passwords required for web sites, safe searches enabled on search engines, and YouTube restricted mode to limit the online moral corruption.

This paper recommends timely provision of accurate and comprehensive information and life skills training regarding sexual and reproductive health and rights (SRHR) for adolescents to achieve sexual health and rights and avoid negative health outcomes such as unplanned pregnancies, high HIV/AIDs new infections, and other negative sexual practices.

Topics such as puberty and reproductive development are presented in upper primary or lower secondary levels after most students have already gone through puberty. Much of the content pertaining to pregnancy prevention, including condoms negotiation and use, other relevant contraceptives methods, delay of sex, and abstinence are partly covered in secondary school level. This is under the presumption that the content is more age appropriate at this level; low transition rates into secondary school, exposure to sexual intercourse earlier before joining secondary school, or dropping out of school at early stages preclude many adolescents from the benefits of sexuality education. The timing, consistence, and continuity of these contents within the various curricula should be addressed.

In Kenya, teachers face pressure to focus on examinable subjects, often at the expense of (LSE). Evaluations of (LSE) programmes have revealed that teachers are often inadequately trained to deliver the lessons and may feel uncomfortable discussing sexual issues. Thus, they may not teach them or schools may encounter resistance from religious groups when addressing sexual education that deviates from a conservative and abstinence-only approach. Evaluation of LSE implementation should therefore be done at a national level in Kenya to determine the extent to which the various curricula are actually being taught.

\section{Appendix}

\section{$\mathbf{A}$}

A.1. LaSalle's Invariance Principle. LaSalle's invariance principle is useful in proving global asymptotic stability for continuous and discrete dynamical systems.

Definition A.1 (invariant set). A set $\Psi$ is said to be invariant with respect to the system of ordinary differential equations $\dot{x}=f(x)$ if all trajectories $\mathrm{x}(\mathrm{t})$ that originate from the set do not leave the set $\Psi$ at any time.

Theorem A.1 (LaSalle's invariance principle). Suppose the set $\Psi \subset D$ is compact and positively invariant with respect to the system of ordinary differential equations $\dot{x}=f(x)$. Suppose further that the function $V$ defined by $V: D \longrightarrow \mathscr{R}$ is continuously differentiable and that $\dot{V} \leq 0$ on $\Psi$. If $E^{*}=x \in \Psi: \dot{V}(x)=0$ and $E^{*}$ is the largest invariant set in $E$, then every solution of the system of ordinary differential equations $\dot{x}=f(x)$ that begins in the set $\Psi$ approaches $E^{*}$ as time $t$ approaches infinity.

\section{A.2. Stable Solution}

Definition A.2 (stable solution). A solution $x^{*}(t) \in \mathscr{R}^{n}$ of the dynamical system (3) is said to be stable if given $\varepsilon>0, \ni \delta\left(\varepsilon, t_{0}\right)>0$ such that for $t>t_{0}$ and for any neighboring solution $x(t) \in \mathscr{R}^{n}$,

$$
\left\|x(t)-x^{*}(t)\right\|<0
$$

whenever

$$
\left\|x\left(t_{0}\right)-x^{*}\left(t_{0}\right)\right\|<\delta[21]
$$

The solution $x^{*}(t) \in \mathscr{R}^{n}$ of a dynamical system is said to be locally stable if neighboring trajectories or solutions that originate close to $x^{*}(t)$ stay close it over time. The solution $x^{*}(t) \in \mathscr{R}^{n}$ is locally asymptotically stable if it is locally stable and slight or infinitesimal small perturbations from it decay to zero over time. Additionally, the solution $x^{*}(t) \in \mathscr{R}^{n}$ is globally asymptotically stable if it is stable and any perturbations from the solution decay to zero or if trajectories originating from any initial data eventually relax to the solution.

\section{Data Availability}

The data supporting this model are from published articles and are cited at relevant places. 


\section{Conflicts of Interest}

The authors declare that they have no conflicts of interest regarding the publication of this paper.

\section{Acknowledgments}

The authors sincerely acknowledge the Department of Mathematics, Masinde Muliro University of Science and Technology, for the support while undertaking this research.

\section{References}

[1] A. O. Binuyo, "Adeyemi olukayode binuyo eigenvalue Elasticity and sensitivity Analyses of the transmission dynamic Model of corruption," Journal of the Nigerian Society of Physical Sciences, vol. 1, pp. 30-34, 2019.

[2] Kenya Demographic and Health Survey, 2014.

[3] Aids Control Council Report, 2018.

[4] National AID. and STI's Control Programme Report, Nascop, 2018.

[5] J. Lema, Study to Determine The Knoweldge, Alttitude and Use of Contraceptives with Relationships to Sexual Knoweledge and Behaviours Among Adolescent Secondary School Girls in Cosmopolitan City in Africa Unpublished Dessertation, University of Nairobi, Nairobi Kenya, 1987.

[6] J. G. Kigondu, "The organisation of family planning services for adolescent in Kenya J.K.G Mati," 1989.

[7] Ministry Health, National Guideline for Provision of Adolescent and Youth Friendly Services in Kenya (Reprodutive and Meternal Health Service Unit), Ministry Health Kenya, Nairobi, Kenya, 2nd edition, 2016.

[8] World Health Organiation (WHO) Europe and Federal Centre for Health Education (BZgA), Standard for Sexuality in Europe. A Framework for Policy Makers. Educational and Health Authorities and Specialists, WHO Europe and Federal Centre for Health Education(BZgA), Cologne, Germany, 2010.

[9] T. A. Lambo, Social And Health Problems of Adolescents in Transitional Cultures of Africa Presented at a Meeting of the World Health Organisation Experts Committee on Health Problem of Adolescent Geneva, Eastern African literature Bureau, Nairobi, Kenya, 1977.

[10] C. M. Worthman and J. W. M. Whiting, "Social changes in adolescent sexual behaviours, mate selection and primarital pregnancy rates in a Kikuyu community Ethos 15:145-165, 1987 and J.D Herzog Initiation and high school in developing of a kikuyue lf-concept," 1973.

[11] S. Abdulrahman, "Stability analysis of the transmission dynamics and control of corruption' Pacific," Journal of Science and Technology, vol. 15, no. 1, pp. 99-113, 2014.

[12] S. Athithan, M. Ghosh, and X.-Z. Li, "Mathematical modeling and optimal control of corruption dynamics," Asian-European Journal of Mathematics, vol. 11, no. 6, Article ID 1850090, 2018.

[13] O. M. Nathan and K. O. Jackob, "Stability analysis in a mathematical model of corruption in Kenya," Asian Research Journal of Mathematics, vol. 28, pp. 1-15, 2019.

[14] L. Lemecha, "Modelling corruption dynamics and its analysis," Ethiopian Journal of Sciences and Sustainable Development, vol. 5, no. 2, pp. 13-27, 2018.

[15] H. T. Alemneh, "Mathematical modeling, analysis, and optimal control of corruption dynamics," Journal of Applied Mathematics, vol. 2020, Article ID 5109841, 13 pages, 2020.
[16] A. O. Binuyo and V. O. Akinsola, "Stability analysis of the corruption free equilibrium of the mathematical model of corruption in Nigeria," Mathematical Journal of Interdisciplinary Sciences, vol. 8, no. 2, pp. 61-68, 2020.

[17] F. Y. Egudam, F. Oguntolu, and T. Ashezua, "Understanding the dynamics of corruption using mathematical modeling approach," International Journal of Innovative Science, Engineering and Technology, vol. 4, no. 4, pp. 2348-7968, 2017.

[18] O. Danford, "Mark kimathi and silas mirau mathematical modelling and analysis of corruption dynamics with control measures in tanzania," Journal of Mathematics and Informatics, vol. 1, pp. 58-79, 2020.

[19] O. M. Nathan and K. O. Jackob, "Mathematical modeling and analysis of mathematics anxiety behavior on mathematics performance in Kenya," Journal of Advances in Mathematics and Computer Science, vol. 35, no. 4, pp. 46-62, 2020.

[20] D. W. Jordan and P. Smith, "Nonlinear ordinary differential equations," in An Introduction to Dynamical SystemsOxford University Press, New York, NY USA, 1999.

[21] R. M. Anderson, Infectious Diseases of Humans: Dynamics and Control, Oxford University Press, Oxford, England, 2nd edition, 1991.

[22] D. R. Merkin, Introduction to the Theory of Stability, SpringerVerlag, Berlin, Germany, 1997.

[23] J. P. LaSalle, "The stability of dynamical systems," in Proceedings of the Regional Conference Series in Applied Mathematics, SIAM, Philadelphia, PA, USA, April 1976.

[24] J. Carr, Application of Center Manifold Theory, SpringerVerlag, New York, NY, USA, 1981.

[25] C. Castillo-Chavez and B. Song, "Dynamical models of tuberculosis and their applications," Mathematical Biosciences and Engineering, vol. 1, Article ID 361404, 2004.

[26] J. Li, Y. Xiao, F. Zhang, and Y. Yang, "An algebraic approach to proving the global stability of a class of epidemic models, Nonlinear Analysis," Real World Applications, vol. 13, 2016.

[27] W.-W. Shi and Y.-S. Tan, “Transmission dynamics and optimal control of an influenza model with quarantine and treatment," International Journal of Biomathematics, vol. 5, no. 3, 14 pages, Article ID 1260011, 2012.

[28] J. Hofbauer and J. W.-H. So, "Uniform persistence and repellors for maps," in Proceedings of the American Mathematical Society, vol. 107, no. 4, p. 1137, 1989.

[29] G. Butler, H. I. Freedman, and P. Waltman, "Uniformly persistent systems," in Proceedings of the American Mathematical Society, vol. 96, no. 3, p. 425, 1986.

[30] H. Zhang, Y. Li, and W. Xu, "Global stability of an SEIS epidemic model with general saturation incidence," ISRN Applied Mathematics, vol. 2013, Article ID 710643, 11 pages, 2013.

[31] S. M. Blower and H. Dowlatabadi, "Sensitivity and uncertainty analysis of complex models of disease transmission: an hiv model, as an example," International Statistical Review/Revue Internationale de Statistique, vol. 62, no. 2, pp. 229-243, 1994.

[32] C. Nakul, J. M. Cushing, and J. M. Hyman, "Bifurcation analysis of a mathematical model for malaria transmission," SIAM Journal on Applied Mathematics, vol. 67, no. 1, pp. 24-45, 2006.

[33] Kenya National Bureau of Statitics, Kenya National Housing and Population Census, Kenya National Bureau of Statitics, Nairobi, Kenya, 2009. 